\title{
Clinical Performance of High Frequency QRS Analysis for Detecting Ischemia Using a Limited Sampling Rate
}

\author{
Noam Omer ${ }^{1}$, Shimon Abboud ${ }^{2}$, Yair Granot ${ }^{1}$ \\ ${ }^{1}$ Biological Signal Processing, Tel-Aviv, Israel \\ ${ }^{2}$ Tel-Aviv University, Tel-Aviv, Israel
}

\begin{abstract}
In recent years High Frequency QRS analysis (HFQRS) which uses information from the depolarization phase of the cardiac cycle has shown superior accuracy in the detection of stress induced ischemia compared to changes in the repolarization phase. HFQRS analysis typically uses the frequency band of 150-250 $\mathrm{Hz}$ and therefore the default requirement was for the ECG to be sampled at $1 \mathrm{kHz}$. In the present study we examined how HFQRS measurements are affected by a lower sampling rate and evaluated the clinical performance of such analysis.

A group of 174 stress ECG records, which were originally sampled at $1 \mathrm{kHz}$, were analyzed twice using the same HFQRS application: once with the original files and once following decimation to simulate records sampled at $500 \mathrm{~Hz} .17 \%$ of the patients had significant ischemia and the gold standard was determined according to myocardial perfusion imaging (SPECT).

Identical sensitivity of $70 \%$ was achieved for both analyses and statistically insignificant difference in specificity was found $(81 \%$ vs $76 \%, p=0.39$ for the $500 \mathrm{~Hz}$ compared to the original records, respectively).

The results imply that much of the HFQRS essential information for detecting ischemia exists in the limited frequency band and that the diagnostic performance of the $H F Q R S$ are not significantly affected as a result of using a sampling frequency of $500 \mathrm{~Hz}$.
\end{abstract}

\section{Introduction}

Ischemic heart disease (IHD) remains the global leading cause of death, accounting for almost 9 million deaths in 2015 [1]. Given that different treatment options which could substantially reduce mortality are available, an early detection of IHD is of high importance in medicine [2]. ECG Stress Testing (EST) is to date the most common modality for an early diagnosis of IHD. It is mostly used as a gatekeeper for cardiac imaging techniques in intermediate-risk patients mainly due to its availability, safety and low cost. However, it is widely recognized that it suffers from low accuracy with a sensitivity of $45 \% 50 \%$ [3]. In the female population, accuracy is even lower with an increased rate of false positive results [4].

In recent years several clinical studies have shown that High Frequency QRS analysis (HFQRS) is more accurate in the detection of stress induced ischemia compared to conventional ST-segment analysis [5-8]. These studies indicate that the use of HFQRS analysis significantly increases sensitivity and maintains specificity together with a significant contribution to the incremental diagnostic value of the EST. HFQRS has the potential to improve the diagnosis and monitoring of myocardial ischemia and reduce healthcare costs [9]. Furthermore, the American Heart Association included HFQRS analysis in its scientific statement regarding EST standards pointing out that it has been found to have useful test performance characteristics for the detection of coronary artery disease (CAD) [10].

The physiological origin of the HFQRS components is directly related to the nature of the electrical conduction system of the heart and was studied in a mathematical simulation [11], as well as in an animal model [12-13]. With the help of signal processing techniques lowamplitude high frequency signals (measured in $\mu V$ ) can be measured during standard EST and indicate the presence of stress induced ischemia [9]. Unlike conventional ECG analysis, which is based on changes in the repolarization phase of the cardiac cycle (ST segment deviations) HFQRS analysis uses information from the depolarization phase (QRS complex). The independent nature of these two signals make HFQRS a valuable tool in the diagnostic process since it is not only more accurate, but it in fact adds more information that can be used in concert with the information available from the conventional analysis.

HFQRS analysis typically uses the frequency band of $150-250 \mathrm{~Hz}$, which was confirmed to have significant diagnostic value [12]. For this reason most studies have used ECG records with a sampling rate of $1 \mathrm{kHz}$. The fact that most standard ECG devices use a sampling rate of 500 $\mathrm{Hz}$ limited the use of the HFQRS because of the distortion in the upper band of the HFQRS data. In this study we aimed to assess the actual effect of a limited bandwidth on 
the HFQRS measurements and to evaluate how it will affect the diagnostic performance of an HFQRS application.

\section{Methods}

For the investigation we have used EST records which were originally sampled at $1 \mathrm{kHz}$. These records were analyzed twice using the same HFQRS application. Once, with the original $1 \mathrm{kHz}$ files and once following decimation to simulate records sampled at $500 \mathrm{~Hz}$. The down-sampled files were analyzed using an HFQRS application which was adapted to process $500 \mathrm{~Hz}$ signals, but was not modified in any other way. Thereafter, the HFQRS measurements were quantitatively compared and the diagnostic performance of both analyzes were evaluated.

\subsection{Analysis Group}

The analysis group included 174 records of suspected IHD patients who underwent a treadmill EST. Based on the patient's gold standard each record was classified into one of two groups: ischemic and non-ischemic. Gold standard was derived from myocardial perfusion imaging (SPECT) and the criteria for a significant ischemia was determined as follows: patients who had $10 \%$ or more LV ischemia were considered as ischemic and patients who had less than $10 \% \mathrm{LV}$ ischemia were considered as non-ischemic [5]. Accordingly, the ischemic group contained 30 (17\%) patients and the non-ischemic group contained 144 records. All records had a valid HFQRS interpretation for both the $1 \mathrm{kHz}$ and the $500 \mathrm{~Hz}$ versions.

\subsection{HFQRS Analysis Application}

The HFQRS analysis was performed using a designated software (HyperQ, BSP Ltd., Tel-Aviv). This application extracts the HFQRS measurements from EST data and was previously shown to provide valuable diagnostic information for stress induced ischemia [6-8]. Briefly, an averaged QRS complex is calculated after alignment for every time segment over the course of the test. Then, these averaged complexes are digitally filtered to extract the HFQRS frequency band and are thereafter quantified. These measurements are used to create the HFQRS time curve which associates an HFQRS measurement to a specific time segment. From these curves the difference between the maximal and the minimal intensities is derived and used to calculate the absolute and relative reductions for each one of the twelve conventional leads. The criteria for a positive lead (i.e. a lead which indicates stress induced ischemia) is a relative reduction of $\geq 50 \%$ and an absolute reduction of $\geq 1 \mu \mathrm{V}$. For each test one out of three HyperQ interpretations is possible: ischemic, non- ischemic and non-diagnostic. A test with $\geq 3$ positive leads is considered ischemic. A test which complies with one of the following three possibilities is considered nondiagnostic: (1) A test with exactly 2 positive leads and $\geq 1$ noisy lead (i.e. a lead with excessive noise). (2) A test with exactly one positive lead and $\geq 2$ noisy leads. (3) A test without a positive lead and with $\geq 4$ noisy leads. Otherwise, a test is considered non-ischemic.

\subsection{Decimation Process}

In a decimation process we start with a discrete-time signal $x[n]$ and convert it into another discrete-time signal $y[n]$, which consists of sub-samples of $x[n]$. One way to describe a general $\mathrm{M}$-fold decimation process (also known as down-sampling) may be defined by Equation 1 where: $h[k]$ represents a digital anti-aliasing filter and $M$ is the decimation factor. As a results of this process the sampling rate is reduced from $F_{S}$ to $F_{S} / M$.

$$
y[n]=\sum_{k=-\infty}^{\infty} h[k] x[n M-k]
$$

In this study 2 -fold $(\mathrm{M}=2)$ decimation was performed with a rectangular filter kernel, which has sub-optimal frequency response for the transition from $1 \mathrm{kHz}$ to 500 $\mathrm{Hz}$. This kernel may be described by the following impulse response:

$$
h[n]=\frac{1}{2}(\delta[2 n]+\delta[2 n-1])
$$

From Equation 2, it could be noticed that for the derivation of a single sample the selected filter averages every pair of adjacent samples and therefore the filtered signal is down-sampled by 2 .

The decimation itself was used to simulate an antialiasing digital filter which precedes the down sampler to prevent aliasing from occurring during the transition to the lower sampling rate. The bandwidth of the $500 \mathrm{~Hz}$ files (i.e. decimated files) is limited and thus the signal to be processed will contain less energy. Figure 1 shows an example for the spectrum of an averaged high frequency waveform of $1 \mathrm{kHz}$ and $500 \mathrm{~Hz}$ records (dotted-broken and broken lines respectively) together with the HFQRS filter's frequency response (continues line). It can be seen that the upper band of the HFQRS with frequencies around $250 \mathrm{~Hz}$ has less energy compared to the lower part around $150 \mathrm{~Hz}$. This figure demonstrates that although some of the HFQRS signal's energy is lost as a result of the decimation it has only a minor effect on the spectrum of the high frequency waveform within the HFQRS frequency band. 


\section{The Spectrum of High Frequency Waveform}

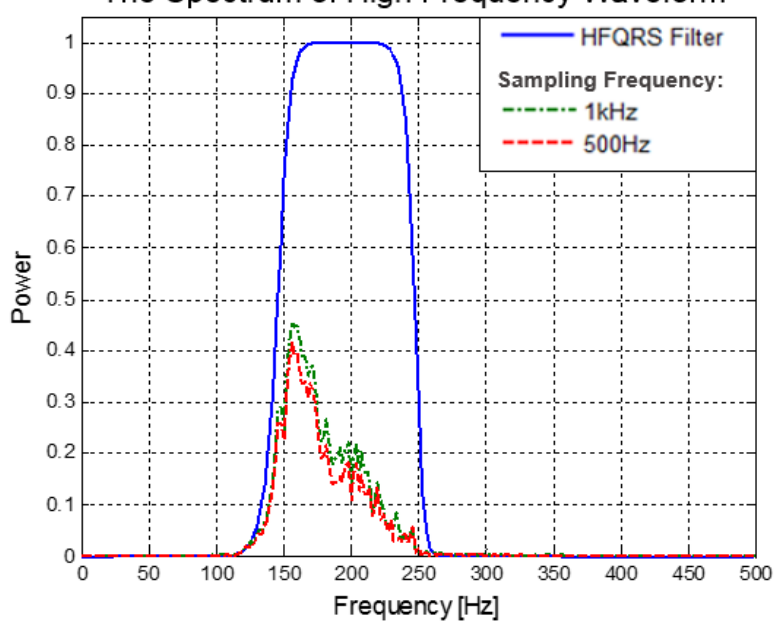

Figure 1. The spectrum of an averaged high frequency waveform of $1 \mathrm{kHz}$ and $500 \mathrm{~Hz}$ records (dotted-broken and broken lines respectively) together with the HFQRS filter's frequency response (continuous line).

\subsection{Evaluation of the HFQRS Results}

In order to quantify the energy loss from the limited sampling rate the differences in the HFQRS measurements were compared between both analyses (in total 2088 HFQRS time curves were used). To assess the differences between individual leads the average number of positive leads in each test was compared. For the evaluation of the clinical performance, sensitivity and specificity were calculated using standard formulas. For the hypothesis testing a two tailed t-test was used and a p-value of $<0.05$ was considered statistically significant.

\section{Results}

The averaged QRS complexes were compared before and after filtration for each analysis. Similar average QRS complexes were found prior to filtration (with negligible differences). This is illustrated at the top of Figure 2 (comparing between (A) and (B)). However, there was an average relative reduction of $15 \%$ (with a standard deviation of $0.5 \%$ ) in the HFQRS amplitude levels between the original files and the $500 \mathrm{~Hz}$ files. An example for this comparison is illustrated at the bottom of Figure 2 which shows a decreased amplitude in the high frequency amplitude between the $500 \mathrm{~Hz}$ record (D) and the original $1 \mathrm{kHz}$ file (C). Moreover, it has been found that this reduction may be approximated by scaling the HFQRS time curves with a scaling factor of 0.85 . Figure 3 shows an example of an original $1 \mathrm{kHz}$ HFQRS time curve (dotted-continuous line), its 0.85 scaled curve (broken line) and its corresponding $500 \mathrm{~Hz}$ curve (continuous line). In this figure the maximal and minimal values are indicated for each curve. As could be seen, in this example both relative reductions are similar (46\% and $47 \%$ ) whereas the absolute reduction of the interpolated $500 \mathrm{~Hz}$ curve is decreased by $14 \%(1.9 \mu$ compared to $2.2 \mu)$. Furthermore, the average number of positive leads per test have shown statistically insignificant differences between both analyses indicating an average number of $3.8 \pm 3.7$ and $3.6 \pm 3.5$ positive leads per test for the $1 \mathrm{kHz}$ and the 500 $\mathrm{Hz}$ records respectively $(\mathrm{p}=0.70)$.

The diagnostic performance comparison have shown identical sensitivity of $70 \%$ for both analyses and a statistically insignificant difference in specificity $(81 \%$ vs $76 \%, \mathrm{p}=0.39$ for the $500 \mathrm{~Hz}$ compared to the original $1 \mathrm{kHz}$ records, respectively).

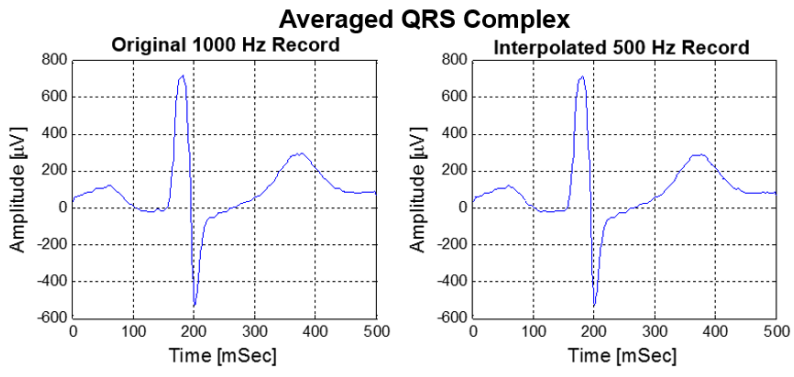

High-Frequency Waveform
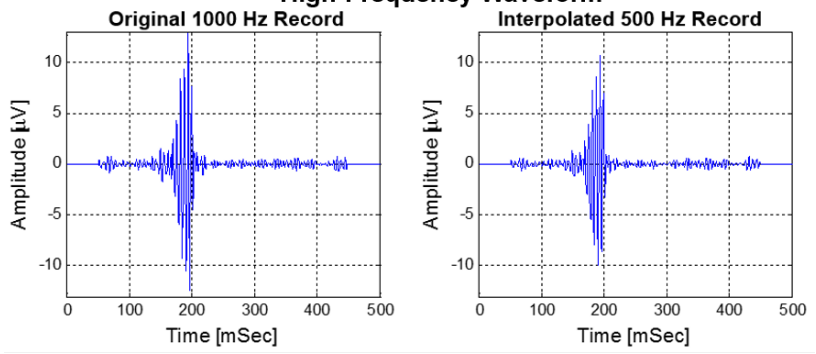

Figure 2. Example for an average QRS complex of an original $1 \mathrm{kHz}$ record and $500 \mathrm{~Hz}$ interpolated record (A and $\mathrm{B}$ respectively) and their corresponding high frequency waveform derived from the filtration to the HFQRS band (C and D respectively).

\section{Discussion}

In this study we examined the effect of a limited sampling rate on the HFQRS analysis by simulating the sampling process of a band limited ECG machine with a digital decimation filter. Although previous studies have found that the $150 \mathrm{~Hz}-250 \mathrm{~Hz}$ band is the most suitable band for HFQRS analysis in EST, the fact that most of the energy in this band is found at the relatively lower frequencies suggests that even a more limited band may still be useful for improving the accuracy of the ubiquitous EST.

When examining the performance of a limited band HFQRS analysis, the first question is what would be the 
result of a straightforward analysis of $500 \mathrm{~Hz}$ signals using the same signal processing adapted to the lower sampling rate. In this preliminary study we have shown that in an initial cohort of typical patients there is no loss of accuracy even when the information found in the upper part of the typical HFQRS band is lost. This result should be tested in a larger and more diverse cohort, but the current study suggests that a possible degradation could be a minor one.

The second question that should be asked in this respect is what would be the optimal manner to analyze lower sampling rate data from EST. The current study has already shown that a decrease of $15 \%$ in the HFQRS intensity exists between the $1 \mathrm{kHz}$ data and the $500 \mathrm{~Hz}$ data. This could be used in order to design a more suitable intensity parameter, for example, by requiring a $0.85 \mu \mathrm{V}$ threshold for defining a positive lead. However, since this threshold depends on the level of noise, it should be analyzed further before determining the optimal value. Nonetheless, future studies should examine general modifications that may be required to account for the known difference between the different sampling rates and their effect on the sampled data.

Further investigation should be focused on the core processes of the HFQRS analysis: the alignment of the QRS complexes, the noise reduction and the differences in maximal and minimal values along the HFQRS time intensity curves. In order to validate the diagnostic performance a further examination on a larger cohort is also required.

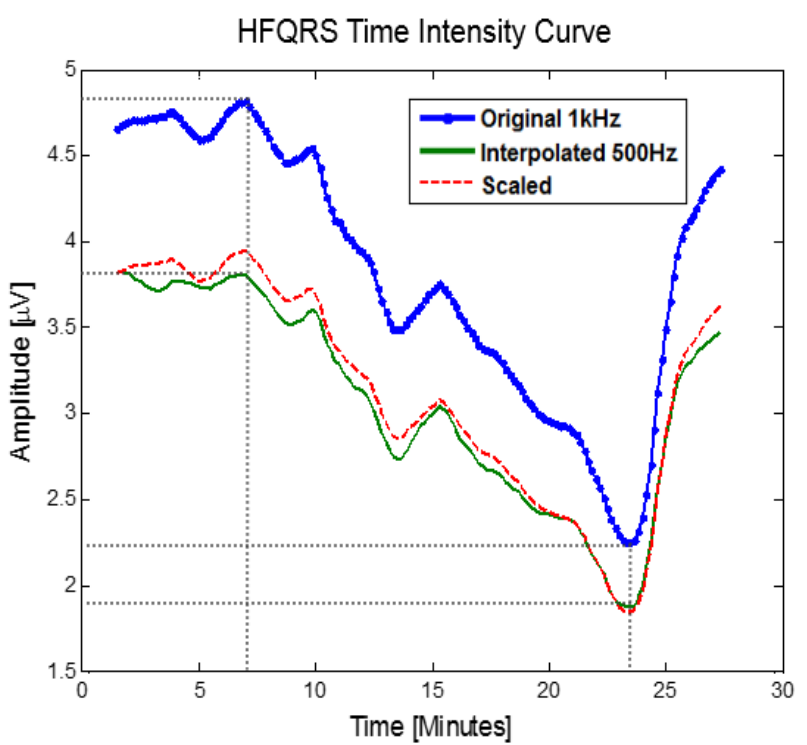

Figure 3. An example of a single original $(1 \mathrm{kHz}) \mathrm{HFQRS}$ time curve (continuous dotted line), its 0.85 scaled curve (broken line) and its corresponding interpolated $500 \mathrm{~Hz}$ curve (continuous line). Maximal and minimal values are indicated for each curve with dotted-faded lines.

\section{Conclusion}

The results of this study imply that significant amount of the essential HFQRS information for detecting ischemia exists in the limited frequency band and that the diagnostic performance of the HFQRS are not significantly affected when using a device with an adequate analog frequency response and sampling frequency of $500 \mathrm{~Hz}$.

\section{References}

[1] WHO at: http://www.who.int/mediacentre/factsheets/ fs310/en. Updated on January 2017.

[2] Fihn S. D., et al. ACCF/AHA/ACP/AATS/PCNA/SCAI/ STS Guideline for the diagnosis and management of patients with stable ischemic heart disease: Executive Summary. JACC 2012;60(24):2564-2603.

[3] Montalescot G., et al. ESC guidelines on the management of stable coronary artery disease. Eur Heart J. 2013;34(38):2949-3003.

[4] Priya Kohli and Martha Gulati, Exercise Stress Testing in Women. Circulation. 2010;122:2570-2580.

[5] Pettersson J., et al. Changes in high-frequency QRS components are more sensitive than ST-segment deviation for detecting acute coronary artery occlusion. J Am Coll Cardiol 2000;36(6):1827-34.

[6] Sharir T., et al. Use of electrocardiographic depolarization abnormalities for detection of stress-induced ischemia as defined by myocardial perfusion imaging. Am. J. Cardiol 2012;109(5):642-50.

[7] Toledo E., et al. Detection of stress-induced myocardial ischemia from the depolarisation phase of the cardiac cyclea preliminary study. J Electrocardiol 2009;42(3):240-7.

[8] Rosenman D., et al. High-frequency QRS analysis improves the specificity of exercise ECG testing in women referred for angiography. J Electrocardiol 2013;46(1):19-26.

[9] Amit G., et al. Quantifying QRS changes during myocardial ischemia: Insights from high frequency electrocardiography. J Electrocardiol. 2014;47(4):505-11.

[10] Gerald F., et al. Exercise Standards for Testing and Training A Scientific Statement From the American Heart Association. Circulation 2013; 128: 873-934.

[11] Abboud S., et al. Simulation of high-resolution QRS complex using a ventricular model with a fractal conduction system. Circulation Research 1991;68;1751: 60.

[12] Abboud S., et al. Detection of transient myocardial ischemia by computer analysis of standard and signal-averaged highfrequency electrocardiograms in patients undergoing percutaneous transluminal coronary angioplasty. Circulation 1987;76(3):585-96.

[13] Mor-Avi V., et al. Effects of coronary occlusion on highfrequency content of the epicardial electrogram and body surface electrocardiogram. Circulation 1987;76(1):237-43.

Noam Omer B.Sc, M.Sc

22a Wallenberg Street, Tel-Aviv, 6971918, Israel

Tel: +97236474840

Fax: +97236471498

Email: noam@bsp.co.il 